\title{
Increase in IL-6 levels among major depressive disorder patients after a 6-week treatment with duloxetine $60 \mathrm{mg} /$ day: a preliminary observation
}

This article was published in the following Dove Press journal:

Neuropsychiatric Disease and Treatment

I0 February 20II

Number of times this article has been viewed

\author{
Michele Fornaro' \\ Matteo Martino' \\ Florinda Battaglia ${ }^{2}$ \\ Salvatore Colicchio ${ }^{3}$ \\ Giulio Perugi ${ }^{4}$ \\ 'Department of Neuroscience, \\ Section of Psychiatry, University \\ of Genova, Genoa, Italy; ${ }^{2}$ Center \\ of Excellence for Biomedical Research \\ (CEBR), Genoa, Italy; ${ }^{3}$ Department \\ of Neurosciences, Catholic University, \\ Rome, Italy; ${ }^{4}$ Department \\ of Psychiatry, Institute of Behavioral \\ Sciences, University of Pisa, Pisa, Italy
}

Correspondence: Michele Fornaro Largo R. Benzi n. 16, Ospedale San Martino, Clinica Psichiatrica Universitaria, Università di Genova, Genoa, Italy-16100

$\mathrm{Tel}+393474 \mid 40003$

Fax +390103537669

Email dott.fornaro@gmail.com
Background: Immune modifications, including changes in interleukin (IL)-6 levels, have often been observed in major depressive disorder (MDD) during treatment with selective serotonin reuptake inhibitors (SSRIs) or the serotonin norepinephrine reuptake inhibitor (SNRI) venlafaxine. Nevertheless, no equivalent observation for the SNRI duloxetine has been made to date.

Method: Sixteen patients diagnosed with MDD and an actual major depressive episode according to $D S M-I V$ criteria and 16 healthy controls entered a 6-week trial with duloxetine $60 \mathrm{mg} /$ day. All subjects $(\mathrm{n}=32)$ were assessed using the Hamilton Depression Rating Scale (HAM-D), the Young Mania Rating Scale (YMRS), and were monitored for IL-6 levels both at baseline and at week 6. Blood samples for IL-6 levels were evaluated by ELISA.

Results: After 6 weeks of treatment, the mean total scores for HAM-D declined both in the depressed and control groups, while IL-6 modification showed an opposite trend both in depressed $(12.38 \pm 19.80$ to $19.73 \pm 18.94 \mathrm{pg} / \mathrm{mL})$ and control subjects $(12.25 \pm 21.12$ to $17.63 \pm 20.44 \mathrm{pg} / \mathrm{mL}$ ), as did YMRS (ns), although none of the subjects switched to (hypo)mania. Of note, IL-6 levels increased significantly only in the responders subgroup $(\mathrm{n}=9 ; P=0.012)$.

Conclusion: The small sample size and weak design of this study limit the validity of our results, which should be regarded as preliminary only. Nonetheless, the trend of increasing IL-6 levels observed in responder patients treated with duloxetine should prompt further controlled, extended studies with larger samples, with the specific aim of better assessing a putative differential role of norepinephrinergic antidepressant stimulation of serotonergic reuptake inhibition in determining modifications in IL-6 levels. Ideally, more accurate replication studies may contribute to further understanding of the complex interaction of mood, antidepressant response, and the immune system.

Keywords: interleukin-6 (IL-6), duloxetine, major depressive disorder (MDD)

\section{Introduction}

In recent years, an increasing number of investigations have explored the relationship between immune and neuroendocrine systems and different psychiatric conditions, especially mood and anxiety disorders, with the aim of improving our understanding their etiopathology and developing more effective therapeutic management. ${ }^{1}$

Neurotransmitters and hormones regulate major immune functions, such as antigen presentation, antibody production, activity, proliferation and traffic of lymphocyte, as well as the process of cytokines secretion, including modulation of T helper (Th) 1 or Th2 cytokine responses. ${ }^{2}$ In the course of inflammatory processes, the activation of the stress system, through induction of a Th2 shift, protects the organism from systemic "overshooting" with Th1/pro-inflammatory cytokines. ${ }^{2}$ Under certain 
conditions, however, stress hormones and the activation of the corticotropin-releasing hormone/substance P-histamine axis may actually promote inflammation, through induction of interleukin (IL)-1, IL-6, IL-8, IL-18, tumor necrosis factor (TNF)-alpha, and C-reactive protein production. ${ }^{2}$ Investigations of immune imbalances in the course of major depressive disorder (MDD) have demonstrated higher serum concentration of some cytokines such as IL-6 (a Th2-type cytokine), IL-1, and TNF $\alpha$ (Th1 cytokines), ${ }^{3-5}$ with similar findings shown also in course of anxious states, ${ }^{6-8}$ reflecting a substantial psychopathological overlap. ${ }^{9}$ Recently, the need for further insights into the antidepressant efficacy of antidepressants and the need for a better understanding of the putative biological bases of depression, prompted correlational studies investigating the influence of antidepressant medications on the immune system, both in psychiatric patients and the general population, and in animals. ${ }^{10}$ To date, most of the immunological investigations carried out in MDD patients treated with antidepressant drugs focused on IL-6 modifications in the course of serotonergic selective serotonin reuptake inhibitors (SSRIs) treatment, which is still the most frequently prescribed class of antidepressants. ${ }^{11}$ Also, while most of the evidence suggests a decrease of IL-6 levels in the course of SSRIs antidepressant treatment, global results are inconclusive, thus raising questions about the relationship between serotonergic-mediated antidepressant response and IL-6 decrease. ${ }^{12}$ Nevertheless, corresponding data for other classes of antidepressant drugs are lacking. Only preliminary evidence is available for the effect of venlafaxine, a serotonin norepinephrine reuptake inhibitor (SNRI), on IL-6 levels during the course of MDD, ${ }^{13}$ with increases or decreases in IL-6 essentially governed by the dose of drug given. ${ }^{13,14}$ Venlafaxine appears to have a complex action on IL-6: administered at low doses, it appears to reduce IL-6 serum levels, but higher doses (eg, $\geq 150 \mathrm{mg} /$ day) seem to induce an increase in IL-6. ${ }^{13,15,16}$ Venlafaxine essentially acts as a pro-serotonergic, SSRI-like, antidepressant at low doses, while at higher doses it also produces a pro-norepinephrinergic effect. ${ }^{17}$ Thus, assessing the effect of venlafaxine on IL-6 should take into account the dose. Remarkably, no equivalent data have been obtained to date for duloxetine, another SNRI drug. Furthermore, duloxetine is supposed to act as a balanced (1:1), pro-serotonergic and pro-norepinephrinergic drug independently on its dosage, ${ }^{18}$ thus IL-6 levels upon duloxetine administration would be expected to follow a linear trend even at low doses (eg, $60 \mathrm{mg}$ /day or lower).
The objective of this study was to investigate the effect of $60 \mathrm{mg}$ /day duloxetine on serum concentration of IL-6 in 16 depressed outpatients and 16 healthy controls within a period of 6 weeks, with the aim of gaining a better understanding of the interaction between antidepressant response and IL-6 levels.

\section{Method}

Twenty outpatients diagnosed with MDD were screened over 12 months for inclusion in a 6-week open-label trial investigating the effect of duloxetine $60 \mathrm{mg}$ /day monotherapy on IL-6 levels. All diagnoses were made according to the Diagnostic and Statistical Manual for Mental Disorders (Fourth Edition) $(D S M-I V)$ criteria $^{19}$ assessed by the Structured Clinical Interview for Axis-I Disorders/Patient edition (SCID-I/P). ${ }^{20}$ At baseline, 16 patients were eligible to enter the study (3 did not sign the informed consent and 1 was coughing), fulfilling all the inclusion criteria, including the actual presence of a single or recurrent, drug-naïve, major depressive episode, age of 18 to 65 years, and able to give a valid informed consent upon approval by the local Ethical Committee. Exclusion criteria included the following: pregnancy, breast-feeding, bipolar disorder, any anxiety disorder, schizophrenia, schizoaffective disorder, dementia, current suicidal and/or psychotic ideation, or concomitant taking of relevant nonpsychotropic medications (including other antidepressants, nonsteroidal anti-inflammatory drugs, and oral contraceptives). Any eventual concomitant medication had to be discontinued at least 15 days prior taking of duloxetine, 4 weeks earlier in case of fluoxetine or depot antipsychotics. Zolpidem 5 to $10 \mathrm{mg} /$ day or diazepam 5 to $10 \mathrm{mg}$ /day were allowed, but could not be taken the night before scheduled assessments. Additional exclusion criteria included history of relevant medical comorbidities (ie, autoimmune, allergic, neoplastic, endocrine diseases, surgery, vascular stroke, or acute or chronic infection within the previous month). A mandatory inclusion criterion was the presence of a baseline total score on the Hamilton Depression Scale (HAM-D) at 17 items $\geq 18^{21}$ (responders would have to show a reduction of HAM-D score at week $6 \geq 50 \%$ vs baseline) when undergoing a physical examination, standard laboratory tests, and immunological monitoring of IL-6. The Young Mania Rating Scale (YMRS) was also administered both at baseline and week 6 in order to assess any eventual drug-induced (hypo)manic switch, defined as a total score $>13 .{ }^{22}$ Blood samples were obtained at 08.30-10.00 AM, in order to limit a potential circadian rhythm variance bias. The whole sample was rescreened at 
week 6 (end of the study) using HAM-D and IL-6 sampling (blood samples were drawn at the same time). Serum levels of IL- 6 were measured using the instant ELISA (enzymelinked immunosorbent assay) method (Bender MedSystems, Burlingame, CA). The same clinical rating scales and laboratory procedures were also applied to the healthy control group $(\mathrm{n}=16)$ defined by a baseline HAM-D score $<7$ (and YMRS < 12). All laboratory procedures were performed at the San Martino Hospital of Genoa, Italy. Over the entire trial, the control group received exactly the same pharmacological regimen (duloxetine $60 \mathrm{mg}$ /day) prescribed to the depressed subjects.

\section{Statistical analysis}

The investigated sample included a low number of subjects. Baseline and week 6 HAM-D and IL-6 variables did not follow a normal distribution according to the Kolmogorov-Smirnov test, therefore requiring nonparametric tests for the comparison of groups in order to assess the hypothetical validity of IL-6 biomarker for antidepressant response to duloxetine. IL-6 levels of responders, nonresponders, and controls were compared using the Mann-Whitney $U$ test.

Pre- and post-treatment IL-6, HAM-D, and YMRS values of the depressed patients and healthy controls were assessed using the Wilcoxon paired samples test. Correlation between IL-6 levels and HAM-D values between responders, nonresponders, and control was assessed using the Pearson correlation coefficient. Two-tailed tests with a $5 \%$ level of significance were used throughout the analyses. All statistical analyses were performed using IBM SPSS Statistics ${ }^{\circledR}$ Version 19 for Windows ${ }^{\circledR}$ (SPSS, Chicago, IL).

\section{Results}

The sample (female, $\mathrm{F}=21$; male, $\mathrm{M}=11$ ) included 16 MDD patients $(\mathrm{F}=12 ; \mathrm{M}=4)$ with a mean baseline HAM-D $=20.94 \pm 2.839$ and YMRS $=2.12 \pm 1.15$ and 16 healthy controls $(\mathrm{F}=9 ; \mathrm{M}=7)$ with a mean HAM-D total score $=3.75 \pm 1.44$ and mean $\mathrm{YMRS}=4.7 \pm 2$.
Mean ages of patients and controls were $51.1 \pm 11$ and $44 \pm 11.6$ years, respectively. No statistically significant differences were observed between the groups for gender distribution, age, or baseline HAM-D (although, by definition, this was higher in patients vs controls). Comparison of mean values of HAM-D and YMRS total scores and IL-6 mean values before and after the treatment in the depressed patients at baseline and control subjects are reported in Table 1 . In the patients group, 14 patients $(87.5 \%)$ showed a reduction in HAM-D total scores after treatment while 2 (12.5\%) were unmodified; IL-6 levels increased in 11 patients $(68.75 \%)$ and decreased in 5 (31.25\%). Notably, in the patients group all clinical modifications in the follow-up observation were significant $(P=0.001)$, but in the nonresponders none of the changes in IL-6 levels were significant. In the control group, $6(37.5 \%)$ subjects showed a reduction in HAM-D scores, 3 (18.75\%) had an increase, and 7 (43.75\%) were unmodified. Six $(37.5 \%)$ subjects reported an increase in YMRS score, 6 $(37.5 \%)$ a reduction, while $2(12.5 \%)$ were unmodified. Eight (50\%) patients showed a reduction in IL-6 levels, 6 (37.5\%) an increase, and 2 (12.5\%) were substantially stable. Notably, none of the modifications observed in the control group was statistically significant, including differences in baseline IL-6 levels between patients and controls. No statistically significant correlations between HAM-D, YMRS scores, and IL-6 levels among responders, nonresponders and controls, before and after treatment, were observed except for the IL-6 levels pre- and post-treatment in responders $(P=0.012)$, as shown in Table 2. The HAM-D $(P=0.001)$ and the YMRS $(P=0.001)$ mean total scores pre- and post-treatment were significantly different between baseline and week 6 in the patients group. No statistical correlations were observed between HAM-D and IL-6 scores at baseline in any group. Finally, none of the subjects enrolled in the study experienced adverse events or significant side effects (eg, nausea, headache) that could be considered as physiological stressors that could have influenced the hypothalamic-pituitary-adrena axis and directly or indirectly affected IL-6 levels.

Table I Mean values of HAM-D and YMRS total scores and IL-6 mean values before and after the treatment in the depressed patients at baseline and control subjects

\begin{tabular}{|c|c|c|c|c|c|c|}
\hline & \multicolumn{2}{|c|}{ HAM-D total score (mean \pm SD) } & \multicolumn{2}{|c|}{ YMRS total score (mean \pm SD) } & \multicolumn{2}{|c|}{ IL-6 levels $(\mathrm{pg} / \mathrm{mL})$ (mean $\pm \mathrm{SD})$} \\
\hline & Pre-treatment & Post-treatment & Pre-treatment & Post-treatment & Pre-treatment & Post-treatment \\
\hline Patients $(n=16)$ & $20.94 \pm 2.84$ & $9.13 \pm 5.8$ & $2.13 \pm 1.15$ & $5.81 \pm 1.80$ & $12.38 \pm 19.80$ & $19.73 \pm 18.94$ \\
\hline Controls $(n=16)$ & $3.75 \pm 1.44$ & $3.5 \pm 1.34$ & $4.69 \pm 2.02$ & $4.75 \pm 2.08$ & $12.25 \pm 21.12$ & $17.63 \pm 20.44$ \\
\hline
\end{tabular}

Notes: In the patient group, the modifications in HAM-D and YMRS scores were highly significant $(P=0.00 \mathrm{I})$ but those in IL-6 levels were not $(P=0.2 \mathrm{I} 5)$. In the control group, there were no significant modifications: HAM-D $(P=0.248)$, YMRS $(P=0.9 .68)$ and IL-6 $(P=0.775)$.

Abbreviations: HAM-D, Hamilton Depression Rating Scale; YMRS, Young Mania Rating Scale; IL-6, interleukin-6. 
Table 2 Modification in interleukin (IL)-6 levels before and after treatment in the responders group $(P=0.012)$ and nonresponders (ns)

\begin{tabular}{lcl}
\hline & IL-6 levels $(\mathrm{pg} / \mathrm{mL})($ mean \pm SD) \\
\cline { 2 - 3 } & Pre-treatment & Post-treatment \\
\hline Responder patients $(\mathrm{n}=9 ; 56 \%)$ & $6.89 \pm 6.878$ & $20.43 \pm 20.32$ \\
Nonresponders $(\mathrm{n}=7 ; 44 \%)$ & $19.45 \pm 28.523$ & $18.83 \pm 18.566$ \\
\hline
\end{tabular}

\section{Discussion}

A number of issues should be considered in the interpretation of our results, which should be considered only preliminary at this time. The open design method of the study and its lack of power, the lack of an accurately matched control group, the unrepresentative sample size, and short follow-up significantly limit the validity of our findings. Additionally, IL-6 serum levels measured during the study might differ from those beyond the blood-brain barrier. Nevertheless, IL-6 levels are "dispersive" in terms of standard deviation even in baseline samples, both in depressed and control subjects, further limiting the statistical validity of our results. Additionally, the unexpected finding of a general increase in IL-6 levels after a 6-week treatment with duloxetine $60 \mathrm{mg} /$ day in responders compared with available data for SSRIs, the lack of larger sampled control groups including other classes of drugs, and/or additional arms with higher doses of duloxetine (eg, $120 \mathrm{mg} /$ day) appear relevant, too.

After 6 weeks of treatment with duloxetine $60 \mathrm{mg} /$ day, all subjects (both patients and controls) showed a decrease in HAM-D total scores and a slight increase in YMRS scores. None of the patients switched to mania according to YMRS cut-off. $^{22}$ Nine of 16 patients had a preliminary response (actually a clinical "remission") and a significant increase in IL-6 levels after 6 weeks.

Although the small sample size of this study might have contributed to almost the lack of statistically significant results, it should be noted that the increase in IL-6 levels after 6 weeks treatment with duloxetine $60 \mathrm{mg} /$ day in responders is an unexpected result. Indeed, the evidence for a modification in IL-6 levels in MDD is still a debated issue. In a pioneering study Maes et al, higher levels of IL-6 levels were observed in patients with MDD vs controls. ${ }^{23}$ Berk et al also found significantly higher levels of IL-6 in patients with MDD. ${ }^{24}$ On the contrary, Kagaya et al found no significant difference in IL-6 levels of depressed patients compared with healthy subjects. ${ }^{25}$ Additionally, Levine et al found decreased levels of IL-6 in the cerebrospinal fluids of depressive patients, ${ }^{26}$ while in a study by Kubera et al, serum IL-6 levels of depressed patients were higher compared with healthy controls. ${ }^{27}$ However, this difference was not statistically significant and the IL-6 levels of treated patients did not decrease upon recovery from depression. ${ }^{27}$ Lanquillon et al found an increase in mean IL-6 levels of responders after antidepressant treatment, while it remained high in nonresponders. ${ }^{5}$ Frommberger et al found a decrease in the IL-6 levels of depressed patients after treatment with antidepressants. ${ }^{28}$ De Berardis et al, ${ }^{12}$ in a systematic review of immunological modulation in the course of antidepressant therapy, including venlafaxine, found that IL-6 levels decreased at low doses (as generally observed in course of treatment with SSRIs), and increased at higher doses. This may be hypothetically due to a differential serotonin/norepinephrine (5-HT/NA) balance caused by the drug, depending on its dosage. Indeed, both 5-HT and NA appear implicated in IL-6 modulation.

These evidences suggested the investigation of duloxetine impact on IL-6 levels, focusing on its pro-norepinephrinergic properties, especially considering that current literature data on other (also) pro-norepinephrinergic drugs is almost unavailable. Specifically, in relation to levels of cytokines and their modulation after treatment with tricyclic antidepressants (TCAs), some studies showed that this class of drugs inhibits the IL-1 $\beta$, IL-2, TNF-alpha, and INF- $\gamma$ (Th1-type cytokines) $)^{29,30}$ and may enhance the production of IL-10 (Th2-type cytokines). ${ }^{31}$ Yet, studies on TCAs and IL-6 show discordant results: while a clinical study reported an increase in IL- $6,{ }^{13}$ other preclinical investigations do not. ${ }^{32,33}$ Furthermore, while TCAs may significantly differ from each other in terms of prevalent stimulation (eg, tertiary amines may promote 5-HT stimulation while secondary ones may enhance the NA-ergic transmission), the investigation of the effect of TCAs on IL-6 levels appears further complicated by the fact that these compounds have a wide range of pharmacodynamic effects. Among other effects, TCAs show muscarinic (M1), adrenergic (alpha-1), and histaminergic (H1) blockade, potentially affecting the immune modulation. Also, it should be noted that desipramine, a secondary amine, inhibits TNF-alpha production, increasing the release of IL-10 and shifting the immune balance toward a Th2-like response. ${ }^{34}$ Regrettably, no equivalent data are available for most of the newer pro-NA-ergic antidepressants such as atomoxetine, or the studies did not focus on IL-6, as in the reboxetine nonclinical studies. However, a single study in animal cells and under specific conditions, reported that pretreatment with reboxetine inhibited the increase of IL-6 due to INF-gamma exposure in the murine microglial cells. ${ }^{35}$ Anyway, it should be remarked that the disparity of results from pre-clinical vs clinical studies might be due to dose 
and timing issues, further requiring rigorous investigations focusing on glucocorticoid-cathecolamine interactions.

Recent evidence indicated that both glucocorticoids and catecholamines (epinephrine and NA) systematically mediate a Th2 shift suppressing role on Th1-cytokine (IL-12 and IFN-gamma) and upregulation of Th2-cytokine production (IL-6 and IL-10) through stimulation of cytoplasmic-nuclear glucocorticoid receptors and $\beta_{2}$ adrenergic receptors, respectively. ${ }^{36}$ Therefore, since catecholamines upregulate IL-6 production, the chronic hyper-noradrenergic state, essentially promoted by NA-ergic stimulation, may drive the increase in systemic IL-6 levels. ${ }^{2}$

Interestingly, baseline IL-6 levels in patients in treatment remission after 6 weeks were considerably higher than in those ending treatment as nonresponders. Nonetheless, since our samples were not statistically representative and ranges of IL-6 levels are very wide, prudence is needed in interpreting our results. Nonetheless, it should be hypothesized that depressed patients who will remit on $60 \mathrm{mg}$ /day duloxetine for 6 weeks (clinically indistinguishable at baseline from those who will not) may exhibit lower levels of IL-6 at baseline which in turn may "predict" a favorable response to treatment with duloxetine $60 \mathrm{mg}$ /day for 6 weeks. It could be therefore hypothesized that the presence of lower levels of IL-6 at baseline among depressed (but not healthy controls) may be due to hypo-noradrenergic activity which may preferentially benefit from the pro-norepinephrinergic action induced by duloxetine, which in turn may "normalize" the IL-6 levels in this subgroup via Th2 shift mediated by NA. Indeed, much prudence as well as larger-sample controlled studies, including proxy measurements of sympatheticnorepinephrinergic activity at baseline and endpoint are warranted prior to extending such hypothesis. Nonetheless, similar findings were already reported by Lanquillon et $\mathrm{al}^{5}$ regarding pre- and post-treatment IL- 6 levels among responders and nonresponders. In this study, the authors found that subjects beginning the treatment in presence of lower baseline IL-6 levels were less prone to significant increase for this parameter after the trial, resembling our finding in that regard, thus suggesting that IL-6 levels "might dichotomize the patients into subsequent responders and nonresponders" prior the initiation of the therapeutic regimen.

If these results could be confirmed by more rigorous investigations, it may be hypothesized that the balance 5-HT/NA may modulate the levels of IL-6. Consequently, IL-6 levels could be considered a potential marker of the Th1/Th2 balance. If this hypothesis can be confirmed by more rigorous study, we could say that the greater the NA-ergic component of antidepressant, the higher the shift of the Th1/Th2 balance toward a Th2-type response.

Elucidating the effect of 5-HT/NA and Th1/Th2 balances on IL-6 levels and demonstrating IL-6 levels as a reliable marker of antidepressant response, may lead to further insights into the understanding of the pathophysiology of depression and antidepressant response even for classes of drugs different from the proven clinically effective, mainly serotonergic agents.

\section{Authors' contributions}

MM conceived the study; MF wrote the main manuscript and performed the statistical analysis. GP and SC served as study consultants. FB carried out the immunological testing. All authors approved the final manuscript.

\section{Disclosure}

The authors received no financial support for this paper and have no conflict of interest to declare.

\section{References}

1. Hickie I, Lloyd A. Are cytokines associated with neuropsychiatric syndromes in humans? Int J Immunopharmacol. 1995;17(8): 677-683.

2. Elenkov IJ. Neurohormonal-cytokine interactions: implications for inflammation, common human diseases and well-being. Neurochem Int. 2008;52(1-2):40-51.

3. Schlatter J, Ortuno F, Cervera-Enguix S. Monocytic parameters in patients with dysthymia versus major depression. J Affect Disord. 2004;78(3):243-247.

4. Maes M. Major depression and activation of the inflammatory response system. Adv Exp Med Biol. 1999;461:25-46.

5. Lanquillon S, Krieg JC, Bening-Abu-Shach U, Vedder H. Cytokine production and treatment response in major depressive disorder. Neuropsychopharmacology. 2000;22(4):370-379.

6. Gill J, Vythilingam M, Page GG. Low cortisol, high DHEA, and high levels of stimulated TNF-alpha, and IL-6 in women with PTSD. J Trauma Stress. 2008;21(6):530-539.

7. Sutherland AG, Alexander DA, Hutchison JD. Disturbance of pro-inflammatory cytokines in post-traumatic psychopathology. Cytokine. 2003;24(5):219-225.

8. Konuk N, Tekin IO, Ozturk U, et al. Plasma levels of tumor necrosis factor-alpha and interleukin-6 in obsessive compulsive disorder. Mediators Inflamm. 2007:65704.

9. Cramer AO, Waldorp LJ, van der Maas HL, Borsboom D. Comorbidity: a network perspective. Behav Brain Sci. 2010;33(2-3):137-150; discussion 150-193.

10. Janssen DG, Caniato RN, Verster JC, Baune BT. A psychoneuroimmunological review on cytokines involved in antidepressant treatment response. Hum Psychopharmacol. 2010;25(3):201-215.

11. Reseland S. Is current prescription practice of SSRI justifiable?. Tidsskr Nor Laegeforen. 2003;123(18):2621.

12. De Berardis D, Conti CM, Serroni N, et al. The effect of newer serotonin-noradrenalin antidepressants on cytokine production: a review of the current literature. Int J Immunopathol Pharmacol. 2010;23(2): 417-422.

13. Kubera M, Kenis G, Bosmans E, et al. Stimulatory effect of antidepressants on the production of IL-6. Int Immunopharmacol. 2004;4(2): 185-192. 
14. Kubera M, Lin AH, Kenis G, Bosmans E, van Bockstaele D, Maes M. Anti-Inflammatory effects of antidepressants through suppression of the interferon-gamma/interleukin-10 production ratio. J Clin Psychopharmacol. 2001;21(2):199-206.

15. Vollmar P, Haghikia A, Dermietzel R, Faustmann PM. Venlafaxine exhibits an anti-inflammatory effect in an inflammatory co-culture model. Int J Neuropsychopharmacol. 2008;11(1):111-117.

16. Piletz JE, Halaris A, Iqbal O, et al. Pro-inflammatory biomakers in depression: treatment with venlafaxine. World J Biol Psychiatry. 2008: $1-11$.

17. Debonnel G, Saint-Andre E, Hebert C, de Montigny C, Lavoie N, Blier P. Differential physiological effects of a low dose and high doses of venlafaxine in major depression. Int J Neuropsychopharmacol. 2007; 10(1):51-61.

18. Trivedi MH, Desaiah D, Ossanna MJ, PritchettYL, Brannan SK, Detke MJ. Clinical evidence for serotonin and norepinephrine reuptake inhibition of duloxetine. Int Clin Psychopharmacol. 2008;23(3):161-169.

19. American Psychiatric Association. Diagnostic and Statistical Manual for Mental Disorders. 4th ed. Washington: American Psychiatric Association; 1994.

20. Ventura J, Liberman RP, Green MF, Shaner A, Mintz J. Training and quality assurance with the Structured Clinical Interview for DSM-IV (SCID-I/P). Psychiatry Res. 1998;79(2):163-173.

21. Hamilton M. A rating scale for depression. J Neurol Neurosurg Psychiatry. 1960;23:56-62.

22. Young RC, Biggs JT, Ziegler VE, Meyer DA. A rating scale for mania: reliability, validity and sensitivity. Br J Psychiatry. 1978;133: 429-435.

23. Maes M, Scharpe S, Meltzer HY, et al. Relationships between interleukin-6 activity, acute phase proteins, and function of the hypothalamic-pituitary-adrenal axis in severe depression. Psychiatry Res. 1993;49(1):11-27.

24. Berk M, Wadee AA, Kuschke RH, O’Neill-Kerr A. Acute phase proteins in major depression. J Psychosom Res. 1997;43(5):529-534.

25. Kagaya A, Kugaya A, Takebayashi M, et al. Plasma concentrations of interleukin-1beta, interleukin-6, soluble interleukin-2 receptor and tumor necrosis factor alpha of depressed patients in Japan. Neuropsychobiology. 2001;43(2):59-62.
26. Levine J, Barak Y, Chengappa KN, Rapoport A, Rebey M, Barak V. Cerebrospinal cytokine levels in patients with acute depression. Neuropsychobiology. 1999;40(4):171-176.

27. Kubera M, Kenis G, Bosmans E, et al. Plasma levels of interleukin-6, interleukin-10, and interleukin-1 receptor antagonist in depression: comparison between the acute state and after remission. Pol J Pharmacol. 2000;52(3):237-241.

28. Frommberger UH, Bauer J, Haselbauer P, Fraulin A, Riemann D, Berger M. Interleukin-6-(IL-6) plasma levels in depression and schizophrenia: comparison between the acute state and after remission. Eur Arch Psychiatry Clin Neurosci. 1997;247(4):228-233.

29. Kenis G, Maes M. Effects of antidepressants on the production of cytokines. Int J Neuropsychopharmacol. 2002;5(4):401-412.

30. Xia Z, DePierre JW, Nassberger L. Tricyclic antidepressants inhibit IL-6, IL-1 beta and TNF-alpha release in human blood monocytes and IL-2 and interferon-gamma in T cells. Immunopharmacology. 1996; 34(1):27-37.

31. Maes M, Song C, Lin AH, et al. Negative immunoregulatory effects of antidepressants: inhibition of interferon-gamma and stimulation of interleukin-10 secretion. Neuropsychopharmacology. 1999;20(4): 370-379.

32. Budziszewska B, Basta-Kaim A, Kubera M, et al. Effect of lipopolysaccharide and antidepressant drugs on glucocorticoid receptor-mediated gene transcription. Pharmacol Rep. 2005;57(4):540-544.

33. Wang W, Danielsson A, Svanberg E, Lundholm K. Lack of effects by tricyclic antidepressant and serotonin inhibitors on anorexia in MCG 101 tumor-bearing mice with eicosanoid-related cachexia. Nutrition. 2003;19(1):47-53.

34. Shen Y, Connor TJ, Nolan Y, Kelly JP, Leonard BE. Differential effect of chronic antidepressant treatments on lipopolysaccharideinduced depressive-like behavioural symptoms in the rat. Life Sci. 1999;65(17):1773-1786.

35. Hashioka S, Klegeris A, Monji A, et al. Antidepressants inhibit interferon-gamma-induced microglial production of IL-6 and nitric oxide. Exp Neurol. 2007;206(1):33-42.

36. Elenkov IJ, Chrousos GP. Stress Hormones, Th1/Th2 patterns, Pro/Anti-inflammatory Cytokines and Susceptibility to Disease. Trends Endocrinol Metab. 1999;10(9):359-368.
Neuropsychiatric Disease and Treatment

\section{Publish your work in this journal}

Neuropsychiatric Disease and Treatment is an international, peerreviewed journal of clinical therapeutics and pharmacology focusing on concise rapid reporting of clinical or pre-clinical studies on a range of neuropsychiatric and neurological disorders. This journal is indexed on PubMed Central, the 'PsycINFO' database and CAS, and is the official

\section{Dovepress}

journal of The International Neuropsychiatric Association (INA). The manuscript management system is completely online and includes a very quick and fair peer-review system, which is all easy to use. Visit $\mathrm{http}: / /$ www.dovepress.com/testimonials.php to read real quotes from published authors. 